Proc. Indian Acad. Sci., Vol. 87 A, (E \& P Sciences-4), No. 11, November 1978, pp. 271-281, (C) printed in India

\title{
Relative performance of different triangular networks in locating regional seismic sources
}

\author{
S K ARORA, T G VARGHESE* and T K BASU \\ Seismology Section, Bhabha Atomic Research Centre, Bombay 400085
}

MS received 13 April 1978; revised 2 September 1978

\begin{abstract}
In regional seismic surveys use of small semipermanent networks of detectors is often made. This study aims at investigating qualitatively the location capability of networks constituted by four (bare minimum) sensors, arranged in different triangular configurations, which may be deployed in a region of known crustal structure. Their relative performances are evaluated on the basis of overall shifts in hypocentre, epicentre and origin time, these being computed by relocating a large number of assumed sources at three representative depths $(0,5$ and $10 \mathrm{~km})$ using an iterative method. Eight constructed examples, of which five pertain to sources lying outside a network and three pertain to sources contained within the outer boundaries of a network, provide data for the study. The influence of shape and size of a given network, on hypocentral determination, relative to the overall dimensions of the expected source region has been discussed.

It is shown that a right-angled triangle (three stations at the vertices and one near the centroid) with its equal orthogonal arms comparable in length with radial distance to epicenters is the best suited configuration for locating sources surrounding the network. Reducing network aperture is found to render the network performance poor. However, if the sources happen to be situated inside the network, the response of one type of network is not appreciably different from that of another but comparatively much better than that of any of the 'source-outside' configurations. Nevertheless, a right-angled triangle (three stations at the vertices and one near the centroid) enclosing the sources seems to be a marginally better choice. Notwithstanding network geometry, an increase in the number of detectors brings about a definite improvement in the estimates of source parameters. This is demonstrated by taking six stations instead of four. It is also shown that network performance remains practically invariant with shallow source depth. The location errors are inferred to be mainly due to the effect of crustal layering.
\end{abstract}

Keywords. Triangular seismic networks; regional sejsmic sources.

\section{Introduction}

The problem of determination of hypocentres of earthquakes using $P$-arrivals at a number of well distributed stations has been tackled as a classical problem in seismology by several workers in the past (Bolt 1960; Herrin et al 1962; Gunst and Engdahl 1962; Engdahl and Gunst 1966; Douglas 1967). In the near field or at short epicentral distances (epicentre within a few hundred kilometers) particularly, use of $P$. and $S$-wave data from small local networks has been suggested by many authors such as Flinn (1960), Nordquist (1962), James et al (1969), Blake and Leighton (1970), Leighton and Blake (1970), Hanks et al (1971), and Ward and Bjornsson (1971).

\footnotetext{
*Deceased on 14th November 1977 at Manila in a fire accident when he was on a UNDP deputation.
} 
In this connection a method based on solving travel-time equations, derived from the geometric ray paths in a known structure and modified by time-terms at each station, has been developed by Crampin (1970). On the other hand Bolt (1970) has discussed a generalised inverse matrix solution which leads to the estimates of location parameters. Westphal and Lange (1967) have described a method of studying microseismicity on a regional scale with special reference to the Fairview Peak area in Nevada. It makes use of $P$ as well as $S$ data from four stations lying approximately in one plane and assumes average propagation speeds of $\boldsymbol{P}$ and $\boldsymbol{S}$ waves. Later on, they (Westphal and Lange 1970) employed this method to determine the sequence of focal points of several Central Nevada microtremors in an attempt to map the geometry of inferred fault in that region.

Originally developed by Bolt and Turcotte (1964) for use within the Berkeley seismographic network, the computer program HYPOLAYR was employed by Eaton et al (1970) for locating more than 600 aftershocks of the 1966 Parkfield-Cholame earthquake in Central California. A slightly modified version of the HYPOLAYR routine was used by Sbar et al (1972) for locating microearthquakes in the Blue Mountain Lake area of the Southern Adirondacks, New York. Crosson (1972) located small earthtremors in the Puget Sound region, Washington, with the help of a non-linear least-squares inversion procedure using $P$-arrivals at more than three stations.

Basically, all source location techniques rest on a common footing of minimising summed squared time-residuals based on a travel-time model. This model is generally deduced by assuming horizontally layered crust with plane parallel layors each of which is characterised by a definite seismic velocity. The iterative adjustment of focal parameters is continued until the sum of squared residuals attain a minimum thereby satisfying convergence criterion. Obviously the location accuracy would largely depend on how accurately the regional travel-times are known. Local variations in crustal structure, if not accounted for, are known to seriously affect source location (Crampin 1970). The probable inaccuracies in the arrival-time measurements and the limited number of observations are other contributory factors. However, biases arising due to unfavourable distribution of stations can also contribute to location errors considerably (Sato and Skoko 1965; Kijko 1977a, 1977b).

In the present study our interest lies in qualitatively investigating the location capability, or what has been called herein as the performance, of different triangular configurations of networks. The nets may be deployed in regions where velocity-depth structure is known. This kind of study becomes increasingly important if the number of receivers is restricted to a bare minimum of four. It is shown, with the help of eight constructed examples, how the geometry and the aperture of a given monitoring network in relation to the overall dimensions of the expected source region influences hypocentral determination. We have also evaluated the relative merits of increasing the number of stations for locating sources. Unfortunately it has not been possible to analytically predict location errors in each individual case. - Nevertheless, from the practical utility point of view, to assess the relative performance of the networks is what has been our aim here.

\section{The method}

The procedure adopted by us involves (i) taking a set of sources whose positions in 
space and time are assumed and then relocating them using expected $P$-onsets at various stations of a given network, and (ii) optimisation of network geometry based on a comparison of sets of calculated shifts in hypocentre, epicentre and origin time. The nature of the present study suggests no usage of any particular travel-time.model, But, for convenience, we have employed $P$-wave travel times available to us from the crustal structure investigations in the vicinity of Gauribidanur in Southern India (Arora 1971, 1975).

In the first part concerning source location we follow the basic approach outlined in a recent paper by Shapira and Bath (1977). These authors were primarily interested in accurately locating rockburst sources (known to exist upto $2000 \mathrm{~m}$ depth) in the Grangesberg iron-ore mining area (about $1.5 \mathrm{~km}$ by $2.5 \mathrm{~km}$ ) in Central Sweden using a small optimal network of geophones. In their method they use a maximum of six detectors. Knowing the actual multi-layered structure, they calculate the arrival times of leading $P$ signal from a few assumed sources. These arrival times are subsequently used to relocate the sources by an iterative procedure based on minimising the residuals. The locations thus obtained are compared with the initially assumed ones and the errors determined. These errors eventually provide clue to the suitability of a network. Although broad principles are the same as stated above, the details of our method are as follows.

Suppose an $M$ th actual source is situated at a point whose true space co-ordinates are $X_{M}, Y_{M}, Z_{M}$. Let it be associated with some known origin time $\left(T_{0}\right)_{M}$. To determine these four source parameters, we assume an initial trial hypocentre to lie at zero focal depth and to have co-ordinates at the earth's surface as given by:

$$
X=\frac{1}{N} \sum_{i=1}^{N} x_{i} ; \quad Y=\frac{1}{N} \sum_{i=1}^{N} y_{i} ; \quad Z=0
$$

where $x_{t}$ and $y_{t}$ represent cartesian co-ordinates (in terms of longitude and latitude respectively) of an $i$ th detector among $N$ detectors.

We now realise a space grid constituted by a number of small parallelopipeds in such a way that they are distributed symmetrically with reference to the initial hypocentre $(X, Y, Z)$ at the centre. Let us assume that this grid structure provides $j$ discrete corners (intersection points) each of which may represent a possible (trial) hypocentre.

Let $T_{i M}$ denote the arrival time, at the $i$ th detector, for a $P$ signal from the $M$ th source. To determine the initial trial origin time $T_{0}$ for this source, we introduce:

$$
T_{0}=T_{i M}^{*}-t^{*}
$$

where $T_{i M}^{*}$ is the minimum of all the $T_{i M}$ 's and $t^{*}$ is the travel time over a distance $R^{*}$. We define $R^{*}$ as the distance of the farthest trial hypocentre, among $i$ trial hypocentres, from the initial point $(X, Y, Z)$. The epicentral distance and the focal depth corresponding to $R^{*}$ can be preset to any suitable values depending upon the extent of the region under investigation (in the present study they are $300 \mathrm{~km}$ and 35 $\mathbf{k m}$ respectively). Similar to the space grid we now form a linear time-grid comprising a series of $k$ equispaced instants of time representing possible (trial) origin times, the initial trial time $T_{0}$ and the time $T_{i M}^{*}$ being the two limits of this grid. 
A search for the best possible combination of the four source parameters is made by computing the minimum of sum of squares of the arrival-time residuals, at various detectors, corresponding to all the available (product of $j$ and $k$ ) trial sources. Thus if $r_{i m}^{2}$ denotes the squared residual at the $i$ th detector, due to an $m$ th trial source, we have:

$$
S_{m}=\sum_{l=1}^{N} r_{i m}^{3}
$$

The optimum solution is represented by that grid point for which $S_{m}$ is minimum. This is obtained first on a coarse space-time grid which is made finer and finer in a progressive manner. The central point of a modified grid is decided by the acceptable solution emerging from the preceding iteration. However, it is a mandatory requirement that the quantity $S_{m}$ should decrease with each iterative step to obtain a convergent solution.

The limit of resolution or, in other words, the grid size can be suitably chosen in each stage of iteration. For the final stage we hold the limit of resolution in epicentre, focal depth and origin time at $0.001^{\circ}$ (about $0.1 \mathrm{~km}$ ), $1 \mathrm{~km}$ and $0.01 \mathrm{sec}$ respectively. It is possible to push these limits further down by making finer subdivisions in the space and time grids so as to realise a nearly continuous function of the four unknowns. However, it is worthwhile seeking resolutions only to the extent that they remain commensurate with the relative accuracies associated with known time-terms in case of any real data.

We found this technique of determining source parameters by the process of refinement through a few iterative steps to be extremely economical in terms of computer time. The relative accuracy of the final solution, however, is a non-linear function of the grid size. For example, in a typical source location using rather wide dimensions of space-time grid as $10 \mathrm{~km}$ cube $-1 \mathrm{sec}$, the computed source position is within about $23 \mathrm{~km}$ of the actual one. By reducing the grid size progressively to half, to one-tenth and then to one-hundredth of the initial size, the source location gradually improves to within about $7 \mathrm{~km}, 4 \mathrm{~km}$ and $2 \mathrm{~km}$ respectively.

In the second part of the study we estimate sets of absolute shifts in hypocentre $(\delta R)$, in epicentre $(\delta D)$ and in origin time $\left(\delta T_{0}\right)$ for each of the assumed sources. This is done by comparing the calculated values of the source parameters with their corresponding true values. Thus, for a given source, if $\delta X, \delta Y$ and $\delta Z$ represent errors in the calculated value of the $X, Y$ and $Z$ co-ordinates respectively, we have:

$$
\left.\begin{array}{l}
\delta R=\left[(\delta X)^{2}+(\delta Y)^{2}+(\delta Z)^{2}\right]^{1 / 2} \\
\delta D=\left[(\delta R)^{2}-(\delta Z)^{2}\right]^{1 / 2}
\end{array}\right\}
$$

The error in focal depth determination may not be separately computed since it is implied in $\delta R$. If the estimates of $\delta R$ and $\delta D$ in a given case are nearly equal it would mean that the contribution from $\delta Z$ is negligible. Different network geometries are tested by relocating a reasonably good number of known sources. Their relative merits are finally assessed on the basis of the overall estimates of $\delta R, \delta D$ and $\delta T_{0}$. The lower the values of these functions the higher naturally is the suitability of a network configuration. 


\section{Constructed examples}

We have constituted eight different types of networks, a complete description of which can be seen in table 1 . Seven of them are 4-element nets where three stations are placed at the vertices of the outer triangle and one station inside it. The remaining one network ( 2 GOS listed at No. 5 in table 1) is augmented to have six stations. This helps us to find out how much is the extra advantage gained by deploying few more stations apart from optimising the network geometry. A circular profile of sources centred at the inner station (a central point in the 2 GOS case) is constructed. The first five configurations listed in table 1 pertain to sources lying outside the networks (the ' OS' series) while the next three pertain to sources contained within the main triangle (the 'IS' series).

Table 1. Code names and description of eight different configurations of stationnetworks and source profiles chosen for the present study
No. Code name
Description

1. EOS-1

2. EOS-2

3. ROS

4. GOS

5. 2 GOS

6. EIS

7. RIS

8. GIS
Three stations at the vertices of an equilateral triangle and one station at the centroid of the triangle; sources lying outside the network along a circular profile with the centre of the epicentral distribution coinciding with the centroid; radial distance of the epicentres equals the network aperture (about $65 \mathrm{~km}$ ).

As in the EOS-1 except that radial distance of the epicentres is three times the network aperture (side length of the network is reduced to one-third of that in the EOS-1; about $22 \mathrm{~km}$ ).

Three stations at the vertices of a right-angled triangle with equal lengths of the orthogonal arms and one station at a point, on the vertical median, close to the centroid of the triangle; sources Iying outside the network along a circular profile with the centre of the epicentral distribution coinciding with the central station; radial distance of the epicentres equals the length of the orthogonal arm (about $65 \mathrm{~km}$ ).

Three stations at the vertices of a general triangle and one station at a point inside the triangle; sources lying outside the network along a circular profile (radius: about $65 \mathrm{~km}$ ) with the centre of the epicentral distribution coinciding with the central station.

Six stations at the vertices of two general triangles each having dimensions as in the GOS, placed close but vertically displaced and inverted with respect to each other; sources lying outside the network along a circular profile (radius: about $70 \mathrm{~km}$ ) with the centre of the epicentral distribution coin-

ciding with a point between the two triangles.

Three stations at the vertices of an equilateral triangle (relatively bigger than that in the EOS-1) and one station at the centroid of the triangle; sources lying inside the main triangle along a circular profile with the centre of the epicentral distribution coinciding with the centroid; radial distance of the epicentres (about $55 \mathrm{~km}$ ) equals one-fifth of the network aperture.

Three stations at the vertices of a right-angled triangle (relatively bigger than that in the ROS) with equal lengths of the orthogonal arms and one station at a point, on the vertical median, close to the centroid of the triangle; sources lying inside the main triangle along a circular profile with the centre of the epicentral distribution coinciding with the central station; radial distance of the epicentres (about $40 \mathrm{~km}$ ) equals one-fifth of the length of the orthogonal arm.

Three stations at the vertices of a general triangle (relatively bigger than that in the GOS) and one station at a point inside the triangle; sources lying inside the main triangle along a circular profile (radius: about $40 \mathrm{~km}$ ) with the centre of the epicentral distribution coinciding with the central station. 
Each complete source profile comprises 72 sources, at angular intervals of $5^{\circ}$, whose cartesian co-ordinates are known from their polar co-ordinates. The depths assumed for these sources fall into three categories, viz. 0,5 and $10 \mathrm{~km}$. At each station of a given network $P$ arrival-times are calculated using the regional travel-time model (Arora 1971, 1975; see also figure 1) and some hypothetical origin time. These expected arrival-times eventually form the input to our source relocation procedure described earlier. The computed hypocentres and origin times readily yield values of shifts in the source parameters in accordance with the formulations mentioned in the previous section.

\section{Results and discussion}

The estimates of relocation errors in hypocentre, epicentre and origin time for the three representative focal depths $(0,5$ and $10 \mathrm{~km})$ obtained for eight different configurations of networks are given in table 2. In each set of these estimates the individual quantity represents the average value along with the standard deviation obtained by relocating all the 72 sources in the azimuthal range between 0 and $2 \pi$.

- A scrutiny of the results obtained for surface-focus sources (available under case 1 in table 2) using the four ' $O S$ ' series of configurations (EOS-1 to GOS) indicates that the hypocentral shift $(\delta R)$ of $1.5 \mathrm{~km}$ with a standard deviation of $4.9 \mathrm{~km}$ (shown as $1.5 \pm 4.9 \mathrm{~km}$; table 2) is minimum for the ROS type (right-angled triangle) of configuration. The epicentral shift $\delta D$ and the shift in origin time $\left(\delta T_{0}\right)$ are also found to be minimum at $1.3 \pm 4.6 \mathrm{~km}$ and $0.06 \pm 0.04 \mathrm{sec}$, respectively, for this confguration. In other words, the ROS configuration appears to be most suitable among

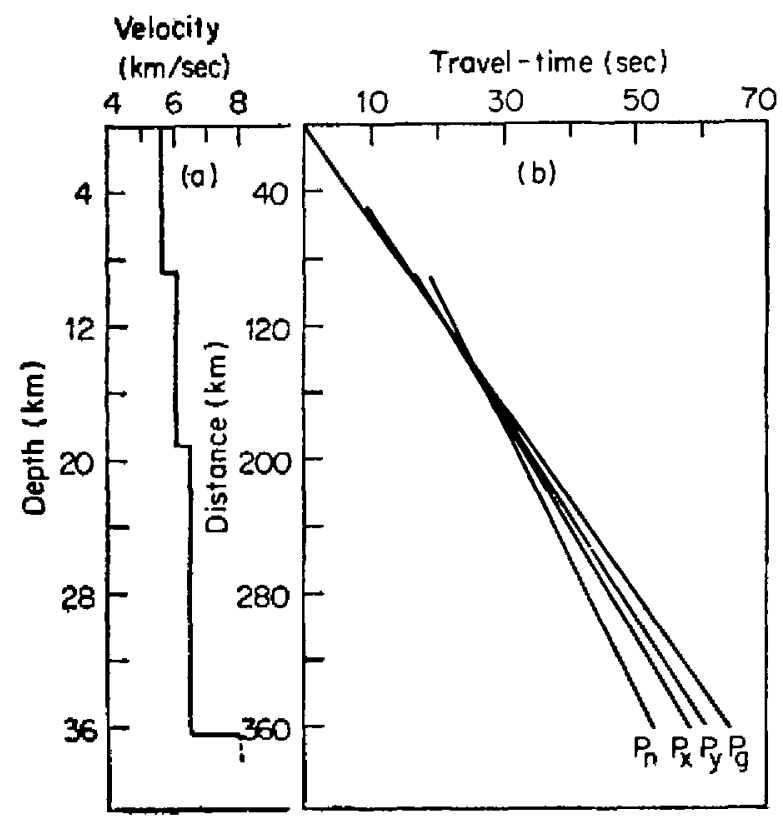

Figure 1. (a) Model of $P$-wave velocity-depth structure used in this study, and (b) typical surface-focus travel times corresponding to this model for different $P$-phases. 
Table 2. Representative estimates of absolute shifts in (a) hypocentre $(\delta R, \mathrm{~km})$, (b) epicentre $(\delta D, \mathrm{~km})$ and (c) origin time $\left(\hat{\delta} T_{0}, \mathrm{sec}\right)$ obtained for three different cases of source depth using eight different network configurations of observing stations

\begin{tabular}{|c|c|c|c|c|c|}
\hline $\begin{array}{c}\text { Network } \\
\text { Configuration } f\end{array}$ & $\begin{array}{l}\text { Actual } \\
\text { souroe } \\
\text { profile } \\
\text { radius } \\
(\mathrm{km})\end{array}$ & Parameter & Case $1^{*}$ & Case $2^{*}$ & Case $3^{*}$ \\
\hline EOS-1 & 65 & $\begin{array}{l}\mathbf{a} \\
\mathbf{b} \\
\mathbf{c}\end{array}$ & $\begin{array}{l}1.9 \pm 5.3 \\
1.5 \pm 4.6 \\
0.08 \pm 0.21\end{array}$ & $\begin{array}{l}1.9 \pm 5.4 \\
1.6 \pm 4.8 \\
0.08 \pm 0.21\end{array}$ & $\begin{array}{l}2.0 \pm 5.6 \\
1.7 \pm 4.9 \\
0.09 \pm 0.21\end{array}$ \\
\hline EOS-2 & 65 & $\begin{array}{l}a \\
b \\
c\end{array}$ & $\begin{array}{l}2 \cdot 5 \pm 4 \cdot 3 \\
2 \cdot 1 \pm 3 \cdot 1 \\
0 \cdot 14 \pm 0 \cdot 26\end{array}$ & $\begin{array}{l}2.7 \pm 3.9 \\
2.4 \pm 3.6 \\
0.17 \pm 0.29\end{array}$ & $\begin{array}{l}3 \cdot 8 \pm 5 \cdot 0 \\
3 \cdot 1 \pm 4 \cdot 4 \\
0 \cdot 22 \pm 0 \cdot 37\end{array}$ \\
\hline ROS & 65 & $\begin{array}{l}a \\
b \\
c\end{array}$ & $\begin{array}{l}1.5 \pm 4.9 \\
1.3 \pm 4.6 \\
0.06 \pm 0.04\end{array}$ & $\begin{array}{l}1.5 \pm 4.8 \\
1.2 \pm 4.6 \\
0.06 \pm 0.04\end{array}$ & $\begin{array}{l}1.6 \pm 4.7 \\
1.3 \pm 4.5 \\
0.06 \pm 0.04\end{array}$ \\
\hline GOS & 65 & $\begin{array}{l}a \\
b \\
c\end{array}$ & $\begin{array}{l}1.8 \pm 4.6 \\
1.7 \pm 4.3 \\
0.06 \pm 0.05\end{array}$ & $\begin{array}{l}1.9 \pm 4 \cdot 5 \\
1.8 \pm 4 \cdot 2 \\
0.07 \pm 0.05\end{array}$ & $\begin{array}{l}1.9 \pm 4.5 \\
1.8 \pm 4.3 \\
0.07 \pm 0.06\end{array}$ \\
\hline $2 G O S$ & 70 & $\begin{array}{l}a \\
b \\
c\end{array}$ & $\begin{array}{l}0.4 \pm 0.4 \\
0.3 \pm 0.3 \\
0.04 \pm 0.04\end{array}$ & $\begin{array}{l}0.5 \pm 0.5 \\
0.4 \pm 0.4 \\
0.05 \pm 0.04\end{array}$ & $\begin{array}{l}0.5 \pm 0.5 \\
0.4 \pm 0.4 \\
0.05 \pm 0.04\end{array}$ \\
\hline EIS & 50 & $\begin{array}{l}\mathrm{a} \\
\mathrm{b} \\
\mathrm{c}\end{array}$ & $\begin{array}{l}0.3 \pm 0.1 \\
0.3 \pm 0.1 \\
0.02 \pm 0.01\end{array}$ & $\begin{array}{l}0.3 \pm 0.1 \\
0.3 \pm 0.1 \\
0.02 \pm 0.02\end{array}$ & $\begin{array}{l}0.3 \pm 0.2 \\
0.3 \pm 0.2 \\
0.02 \pm 0.02\end{array}$ \\
\hline RIS & 40 & $\begin{array}{l}a \\
b \\
c\end{array}$ & $\begin{array}{l}0 \cdot 2 \pm 0 \cdot 1 \\
0.2 \pm 0 \cdot 1 \\
0.01 \pm 0.00\end{array}$ & $\begin{array}{l}0.2 \pm 0.1 \\
0.2 \pm 0.1 \\
0.01 \pm 0.00\end{array}$ & $\begin{array}{l}0.2 \pm 0.1 \\
0.2 \pm 0.1 \\
0.01 \pm 0.01\end{array}$ \\
\hline GIS & 40 & $\begin{array}{l}\mathrm{a} \\
\mathrm{b} \\
\mathrm{c}\end{array}$ & $\begin{array}{l}0.7 \pm 0.5 \\
0.6 \pm 0.4 \\
0.03 \pm 0.03\end{array}$ & $\begin{array}{l}0.6 \pm 0.4 \\
0.5 \pm 0.3 \\
0.03 \pm 0.02\end{array}$ & $\begin{array}{l}0.7 \pm 0.6 \\
0.6 \pm 0.5 \\
0.04 \pm 0.02\end{array}$ \\
\hline
\end{tabular}

†For explanation see table 1.

* Source depth assumed to be $0 \mathrm{~km}$ (Case 1), $5 \mathrm{~km}$ (Case 2) and $10 \mathrm{~km}$ (Case 3).

the four basic configurations. These results are shown in figure 2 which covers onehalf portion of the azimuthal range between 0 and $\pi$. The corresponding picture for the other half between $\pi$ and $2 \pi$ is simply the mirror image of the first half in view of the symmetry about the vertical axis through the centroid of the triangle.

In contrast with the above configuration, the small aperture network EOS-2 (equilateral triangle with side length about $22 \mathrm{~km}$ only) has given values of $\delta R, \delta D$ and $\delta T_{0}$ largest among all the sets at $2 \cdot 5 \pm 4.3 \mathrm{~km}, 2 \cdot 1 \pm 3.1 \mathrm{~km}$ and $0 \cdot 14 \pm 0.26$ sec respectively (table 2). The relatively large errors due to this type of network are indicative of jts poor performance.

The above two sets of results together with those obtained for the EOS-1 and GOS configurations throw some light on the relationship between the performance of a given network and its overall size and shape. It is clear that the influence of increasing 


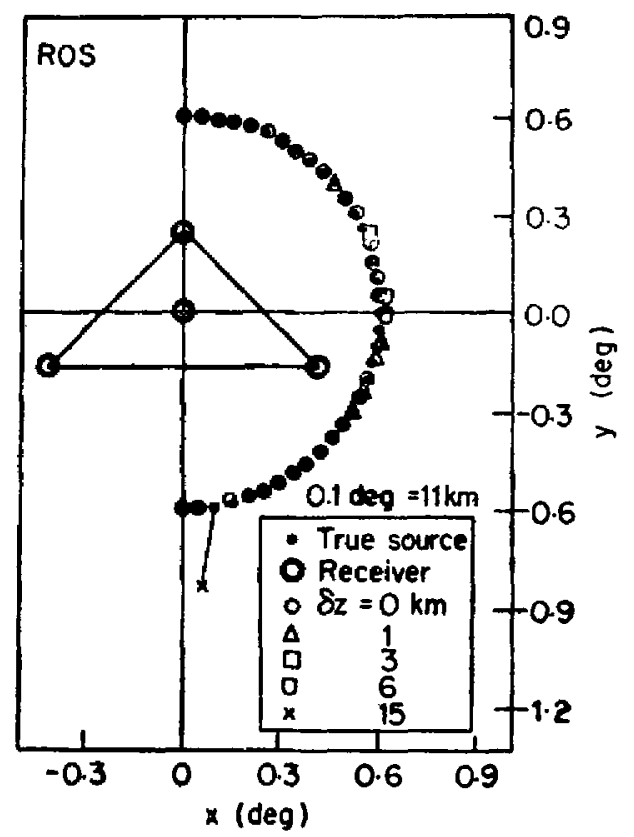

Figure 2. Response of a right-angled triangle type of network having 3 stations at the vertices and 1 near the centroid (ROS; see at No. 3 in table 1) shown for only 37 surface-focus sources lying outside the network and in azimuths between $0^{\circ}$ and $180^{\circ}$ (half side of the symmetrical picture) with respect to the centre of the circular source profile. Hypocentral shifts and focal depth errors for individual sources can be seen from the diagram.

the network aperture to become comparable with epicentral distance is to improve its performance. On the other hand, compacting a network to a size smaller than the radial distance to surrounding epicentres renders its location capability poor. Besides, among the basic triangular shapes of networks, a right-angled triangle (due to its wide aperture effectively) turns out to be the most favourable choice.

Notwithstanding the network geometry, an increase in the number of detectors (for example, use of 6 stations instead of 4 as in the 2 GOS configuration formed by two general triangles placed near each other) brings about a definite improvement in the determination of source parameters. This becomes evident from the set of averages mentioned against the 2 GOS in table 2 and from a complete response shown in figure 3. The typical improvement in this case is about 4 times in source location and about 2 times in origin time compared with the corresponding estimates obtained from the best considered ROS type of network. It would therefore appear that it is increasingly important to choose an appropriate 4-element network if one wants to economise on the number of sensors to reduce both operational problems and cost in the field deployment of surveillance networks.

We now turn to assess the performance of three other configurations belonging to the ' IS ' category in which sources are assumed to be situated inside the outer triangle, By virtue of this condition the network aperture is always larger (5 to 6 times) than the radial distance to epicenters. The pertinent estimates given in table 2 reveal that the response of one type of configuration is not appreciably different from that of 


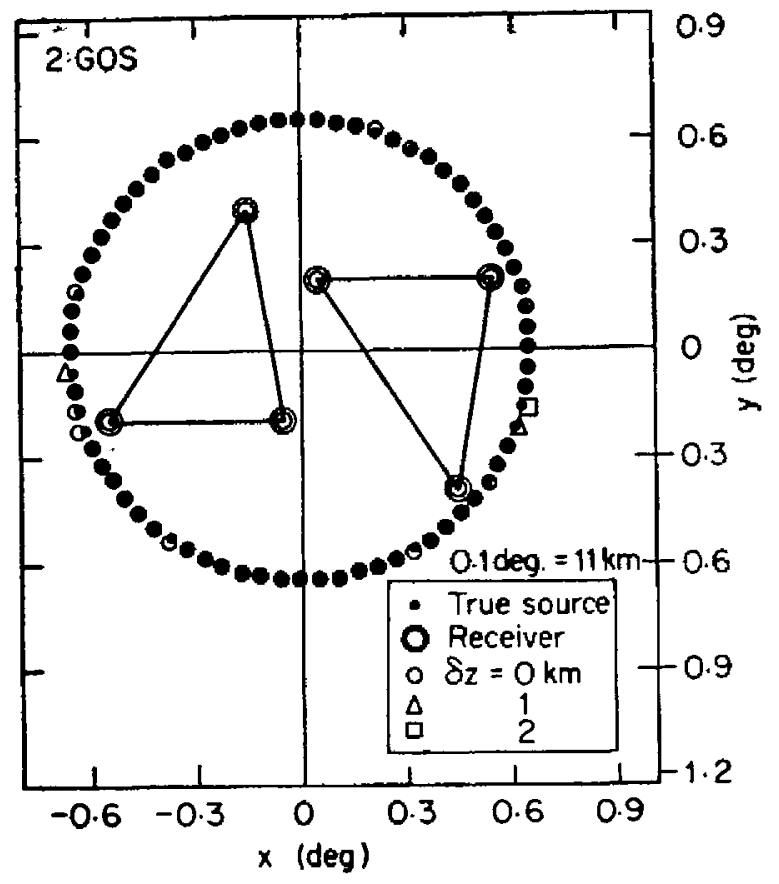

Figure 3. Response of a 6-station network in the form of 2 general triangles each having 3 stations at the vertices ( 2 GOS; see at No. 5 in table 1) shown for 72 surfacefocus sources lying outside the network and in azimuths between $0^{\circ}$ and $360^{\circ}$ with respect to the centre of the circular source profile. Hypocentral shifts and focal depth errors for individual sciurces can be seen from the diagram.

another, but comparatively much better than that of any of the 4-element 'sourceoutside' configurations. Nevertheless the average estimates for the RIS configuration (right-angled triangle once again) are found to be the lowest at $0.2 \pm 0.1 \mathrm{~km}$, $0.2 \pm 0.1 \mathrm{~km}$ and $0.01 \pm 0.00 \mathrm{sec}$ (table 2) corresponding to $\delta R, \delta D$ and $\delta T_{0}$ respectively. This suggests a slightly better suitability of the RIS configuration than the other two. Figure 4 shows the response of this configuration, which is symmetrical about the vertical axis through the centroid as in the two previous cases (ROS and EOS-2).

In the sets of error estimates for both ' $O S$ ' and 'IS' series of configurations (table 2) one notices that the values of $\delta D$ are either somewhat lower than or equal to the corresponding values of $\delta R$. It shows that the various hypocentral shifts are contributed mainly by the epicentral shifts, the corresponding source depths being computed fairly close to their true values.

By taking a typical configuration (general triangle type of network: GOS) we show that, by and large, location errors are mainly due to the effect of crustal layering, those arising on account of our discrete search procedure of relocating sources being restricted to the grid size. For this purpose we assume a homogeneous earth model having a typical signal velocity of $6 \mathrm{~km} / \mathrm{sec}$ (layering virtually removed) and relocate all the 72 surface-focus sources around the network. The average values corresponding to $\delta R, \delta D$ and $\delta T_{0}$ obtained are as small as $1.0 \pm 1.0 \mathrm{~km}, 0.3 \pm 0.3 \mathrm{~km}$ and 0.02 


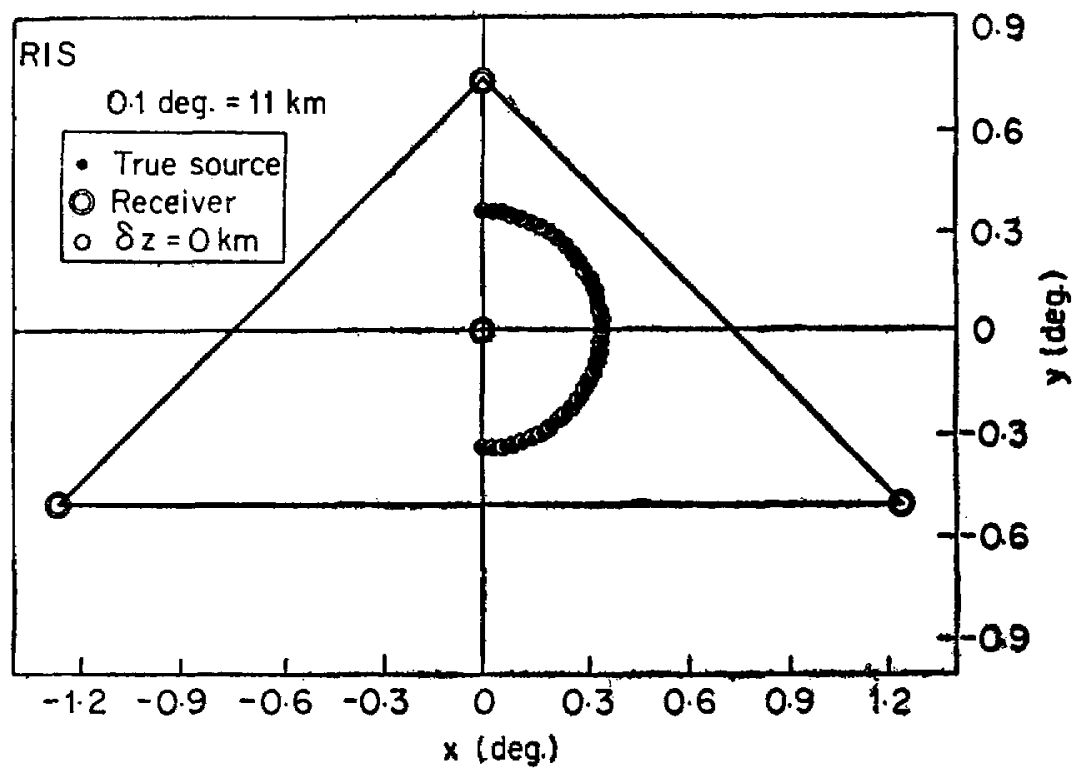

Figure 4. Response of a right-angled triangle type of network having 3 stations at the vertices and 1 near the centroid (RIS; see at No. 7 in table 1) shown for only 37 surface-focus sources contained within the outer boundaries of the network and in azimuths between $0^{\circ}$ and $180^{\circ}$ (half side of the symmetrical picture) with respect to the centre of the circular source profile. Hypocentral shifts and focal depth errors for individual sources can be seen from the diagram.

$\pm 0.02 \mathrm{sec}$, respectively. Hence, although what one generally gets in the form of location errors is a combined of crustal layering and size of the space-time grid used, the influence of the grid size alone (if small enough) is insignificant compared to that due to the layering.

The relative performance of the different networks under condition of increased source depth is demonstrated by repeating the whole exercise with sources at $5 \mathrm{~km}$ and $10 \mathrm{~km}$ depths. The results thus obtained are presented under case 2 and case 3 of table 2 . Obviously there seems to be practically no significant change in the network response if the sources happened to reside at shallow depth. This statement holds good for moderate aperture nets. The performance of the small aperture net is found to deteriorate with increasing source depth (see cases 1 to 3 for EOS-2 in table 2). In other words, for regional seismic reconnaissance, the ROS type of configuration for sources which are expected to be situated outside the network boundaries and the RIS type of configuration for sources contained within the main network would function with uniformly good performances from zero to few kilometers focal depth. Therefore, in planning seismicity surveys in regions of known crustal structure, one may gainfully exploit the properties of these two types of moderate aperture networks thereby meeting the purpose with a bare minimum requirement of deploying only four field stations.

\section{Acknowledgement}

We are thankful to Dr G S Murty for his many helpful suggestions. 


\section{References}

Arora S K 1971 Bull. Seism. Soc. Am. 61671

Arora S K 1975 Geophys. Res. Bull. 137

Blake W and Leighton F 1970 Rock mechanics-theory and practice ed. W H Somerton (New York: Academic Press) 429

Bolt B A 1960 Geophys. J. R. Astron. Soc. 3433

Bolt B A and Turcotte F T 1964 Computers in the mineral industry Part 2 ed. G Parks (Stanford Univ. Publ.) 9561

Bolt B A 1970 Bull. Seism. Soc. Am. 601823

Crampin S 1970 Geophys. J. R. Astron. Soc. 21535

Crosson R S 1972 Bull. Seism. Soc. Am. 621133

Douglas A 1967 Nature London 21547

Eatn J P, O'Neill M E and Murdock J N 1970 Bull. Seism. Soc. Am. 601151

Engdahl E R and Gunst R H 1966 Bull. Seism. Soc. Am. 56325

Flinn E A 1960 Bull. Seism. Soc. Am. 50467

Gunst R H and Engdahl E R 1962 Earthq. Not. 3393

Hanks T C, Jordan T H and Minster J B 1971 US Geol. Surv. Professional Paper 73321

Herrin E, Taggart J and Brown Jr. C F 1962 J. Grad. Res. Cent. 3079

James D E, Sacks I S, Lazo L E and Aparico G P 1969 Bull. Seism. Soc. Am. 591201

Kijko A 1977a Pure Appl. Geophys. 115999

Kijko A 1977b Pure Appl. Geophys. 1151011

Leighton F and Blake W 1970 US Bur. Mines Rep. Invest. 7432 pp 18

Nordquist J M 1962 Bull. Seism. Soc. Am. 52431

Sato Y and Skoko D 1965 Bull. Earthq. Res. Inst. 43451

Sbar M L, Armbruster J and Aggarwal Y P 1972 Bull. Seism. Soc. Am. 621303

Shapira A and Bath M 1977 Seism. Inst. Uppsala Rep. 2/77 (Sweden) pp 24

Ward P L and Bjornsson S 1971 J. Geophys. Res. 763953

Westphal W H and Lange A L 1967 Bull. Seism. Soc. Am. 571279

Westphal W H and Lange A L January 1970 Engg. Mining J. 86 